\title{
Neural-Encoded Fuzzy Models for Load Balancing in 3GPP LTE
}

\author{
Aderemi A. Atayero, Matthew K. Luka and Adeyemi A. Alatishe \\ Department of Electrical \& Information Engineering \\ Covenant University \\ Nigeria
}

\begin{abstract}
Post third generation (3G) broadband mobile networks such as HSPA+, LTE and LTE-Advanced offer improved spectral efficiency and higher data rates using innovative technologies such as relay nodes and femto cells. In addition, these networks are normally deployed for parallel operation with existing heterogeneous networks. This increases the complexity of network management and operations, which reflects in higher operational and capital cost. In order to address these challenges, self-organizing network operations were envisioned for these next generation networks. For LTE in particular, Self-organizing networks operations were built into the specifications for the radio access network. Load balancing is a key self-organizing operation aimed at ensuring an equitable distribution of users in the network. Several iterative techniques have been adopted for load balancing. However, these iterative techniques require precision, rigor and certainty, which carry a computational cost. Retrospect, these techniques use load indicators to achieve load balancing. This paper proposes two neural encoded fuzzy models, developed from network simulation for load balancing. The two models use both load indicators and key performance indicators for a more informed and intuitive load balancing. The result of the model checking and testing satisfactorily validates the model.
\end{abstract}

\section{General Terms}

Access Network, Broadband, Models, Soft computing Wireless communication.

\section{Keywords}

Load balancing, neural network, fuzzy logic, LDI Model, USU Model.

\section{INTRODUCTION}

The third generation project (3GPP) Long Term Evolution (LTE) has the core objective of meeting the increasing performance needs of mobile broadband. To this end, LTE among other things use both micro and femto cells as well as relay nodes to achieve the required increase in capacity $[1,2]$. However, this is at the cost of increased network planning and management complexity. Self-Organizing Networks (SON) operations were introduced to improve overall system performance through efficient operations and maintenance. The main drivers for SON operations in mobile networks include [3]:

- Protecting investment and revenue by minimizing human errors in network operations.
- To manage the complexity and parallel operation of LTE (or other new technologies) with existing 2G/3G networks.

- To minimize the cost of network operation by reducing human participation in design, operation and maintenance of the mobile network.

- Cut down capital expenditure by the optimal use of available resources.

Categorized into functional units, a SON operation can be a self-configuration, self-optimization or self-healing operation. Load balancing self-optimizing whose objective of load balancing is to ensure an equitable distribution of cell load among cells or to transfer part of the traffic from congested cells with the aim of improving the overall system capacity [4, 5]. Load balancing can be realized by optimizing the cell reselection/handover parameters such as hysteresis based on load imbalance between neighboring eNodeBs. In addition to improving the overall system capacity, load balancing is of significant benefit to both network operators and subscribers. Autonomous load balancing minimizes human intervention, which helps reduce both capital and operational cost. It also ensures that network resources are evenly used. Subscribers benefit from load balancing in the form of better service satisfaction.

Load balancing is one of the different types of load management procedures in LTE. The other procedures are: Load rebalancing and overload procedure. Load rebalancing is used for partially or fully offloading a Mobility Management Entity (MME), whereas overload management procedure is used to overcome a sudden surge in the loading. Load balancing can be implemented over either the S1 interface (MME load balancing) or the X2 interface (Radio access network load balancing). MME load balancing ensures the equitable distribution of network traffic among MMEs based on their respective capacities. To this end, the procedure uses the Non-Access-Stratum Node Selection Function (NNSF) associated with each eNodeB for the S1 flex function. Load balancing between eNodeBs is aimed at offsetting local load imbalance between neighboring cells in order to optimize overall system capacity. Throughout this disquisition, the term 'load balancing' will be used to mean load balancing among eNodeBs.

The neighboring eNodeBs periodically exchange load information over the X2 interface to track load imbalance in the network. When the load imbalance among neighboring eNodeBs exceeds a predetermined threshold, the load balancing process is initiated. The most important load information normally used is the radio resource usage, which 
represents the uplink and downlink Physical Resource Block (PRB) usage. Generic measurements representing non-radiorelated resource usage are vital for ensuring that network resources are not overstretched by the load-balancing process. The Transport Network Load (TNL) indicator, Hardware (HW) load indicator, and available capacity for load balancing as a percentage of total cell capacity are some of the key generic measurement parameters used. Where LTE is used for parallel operation with other Radio Access Technologies (RATs), the Cell Capacity Class Value (CCCV), which is a relative capacity indicator, must be taken into consideration for a more informed load balancing. Load balancing is usually executed using handover. However, Handover is a computationally expensive task, thus the handover parameters must be optimized to avoid unnecessary handovers and redirections involved in load balancing. The expected results of load balancing include [6]: 1) increased system capacity; 2) UEs at cell border should handover or reselect a less loaded cell based on the cell's hysteresis; 3) a balanced cell load distribution; and 4) reduced manual intervention in network management and optimization tasks.

Several algorithms and techniques have been proposed for load balancing in 3GPP LTE. In [7], an algorithm to find the suitable handover offset value between the overloaded cell and a possible target cell was proposed. In the preliminary procedures of this algorithm, each eNodeB collects measurement report (Reference Signal Received Power) from the served UEs and available resource reports from neighboring cells. If the virtual load of the selected cell exceeds a certain threshold, it begins to transfer UEs to less loaded target cells. This handover process continues until the virtual load of the sending cell goes below the overload threshold, or the virtual load of the target eNodeB exceeds a certain threshold. Hao Wang et al, proposed a load balancing algorithm that considers heterogeneous services in [8]. In this approach, load balancing was treated as a multi-objective optimization problem, with the aim of optimizing the load (fairness) index of services with quality of service (QoS) requirements. Another objective the algorithm used is to maximize the network utility for Best Effort (BE) services. The optimization problem is subjected to physical resource limits and quality of service demands and then solved using sequential optimization methodology. In order to optimize network throughput, Hao Wang et al proposed another load balancing algorithm for the 3GPP LTE in [9]. The framework of for this approach was premised on a network-wide utility function that balances network throughput and load distribution. In the implementation of the algorithm known as Heaviest-First Load Balancing Algorithm, each cell receive load status information from its neighbors and compare it with a threshold value. When the load of the eNodeB exceeds a threshold value, the algorithm selects a user that will gain more throughput when transferred to a given target eNodeB. This iterative procedure is repeated until the load of the overloaded cell is reduced to a value equal to or below the threshold value. The algorithm also ensures that the heaviest load is considered first for load balancing. The cell individual power offset (CIPO) parameter, was used for controlling handovers needed to achieve equitable load distribution in [10]. Each cell adds the cell power of a user to its power offset value and compares this with the corresponding values of its neighbors to decide whether to handover the user or not. In [11], fuzzy logic controller was envisioned for optimizing handover parameters for adaptive load balancing. Since the task of load balancing is ill defined due to the unpredictable mobility of users and other variable factors, there exist a degree of uncertainty and imprecision that can be capitalized upon for a cost effective load balancing solution. Moreover, the need for human intuition in solving nonlinear problems cannot be overemphasized. Hence this paper presents the application of a neural encoded fuzzy technique; specifically, Adaptive Neuro-Fuzzy Inference System (ANFIS) for load balancing in LTE. Additionally, the models developed use Key Performance Indicators (KPIs) in order to increase system performance, thereby justifying the load balancing process..

\section{MODELLING PARAMETERS}

This work proposes two ANFIS models both of which uses the same load indicators (LIs) but utilize different KPIs for load balancing in 3GPP LTE network. The load indicators are: Virtual load (VL) of eNodeBs and their Overall Load State (OS). The first model is based on a KPI termed Load Distribution Index (LDI) while the second one is premised on the number of unsatisfied users in the cell and is termed Unsatisfied Users (USU) model.

\subsection{Load Indicators}

The load indicators used for the design of the load balancing scheme are: the virtual load overall state of the serving cell. These load indicators can be calculated from the number of physical resource blocks used in an eNodeB. The number of physical resource blocks in turn depends on the throughput of the users in a cell.

\subsubsection{Virtual Load:}

A specified Modulation Coding Scheme (MCS) requires a certain SINR, measured at the receiver of the UE, to operate with an acceptably low Bit Error Rate (BER) in the output data. An MCS with a higher throughput requires a higher SINR to operate. Assuming that the best modulation coding scheme is used for a given SINR, the highest data rate $R(\operatorname{SINR})$ that is achievable is represented by Shannon formula as [12]:

$$
R\left(\operatorname{SINR}_{u}\right)=\log _{2}\left(1+\operatorname{SINR}_{i, u}\right)
$$

In order to achieve better approximations to realistic MCS, the mapping function is scaled by attenuation factor (say 0.75 ) and is bounded by a maximum bitrate $(4.8 \mathrm{bps} / \mathrm{Hz})$ and minimum required SINR $(-6.5 \mathrm{~dB})$. The mappings of certain physical channels to resource elements are described by the use of Resource Blocks. The scheduler in the eNodeB allocates resource blocks to users for a specified amount of time. A physical resource block (PRB) is defined as $N_{s c}^{R B}$ consecutive carriers in the frequency domain and $N_{\text {symb }}^{D L}$ consecutive OFDM symbols in the time domain [13]. A PRB consists of $N_{\text {symb }}^{D L} \times N_{s c}^{R B}$ resource elements corresponding to $180 \mathrm{KHz}$ in the frequency domain and one slot in the time domain. The physical resource blocks corresponding to the various downlink bandwidths supported in LTE are given in Table 1.

Table 1 PRBs of different downlink bandwidths.

\begin{tabular}{|l|l|l|l|l|l|}
\hline Bandwidth (MHz) & 1.4 & 3.0 & 5.0 & 10 & 20 \\
\hline $\begin{array}{l}\text { Physical Resource } \\
\text { Blocks }\left(\boldsymbol{N}_{\boldsymbol{t}}\right)\end{array}$ & 6 & 15 & 25 & 50 & 100 \\
\hline
\end{tabular}


The amount of PRBs required by a user can be expressed as [14]:

$$
N_{u}=\frac{D_{u}}{R(S I N R)_{u} \cdot B W}
$$

Where: $D_{u}=$ required data rate and $\mathrm{BW}$ is the transmission bandwidth of one resource blocks (180 kHz for LTE) and $R(\operatorname{SINR})_{u}$ is the achievable throughput. Fig. 1 presents the relationship between SINR and the number of resource block required for downlink transmission. A number of services and applications such as VoIP and IPTV require a certain quality of service (QoS) level. For instance, constant bit rate services are only satisfied if they get a certain bit rate $D_{u}$. For this work, a homogenous constant bit rate of $1 \mathrm{Mbps}$ is assumed for all users.

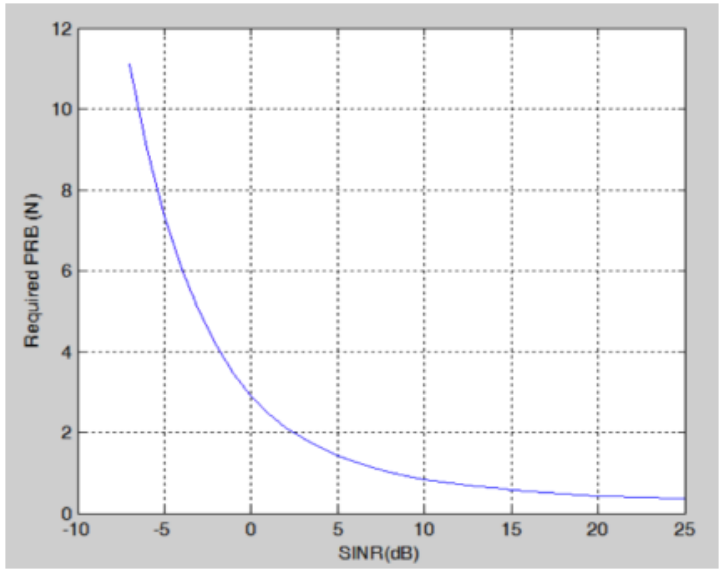

Fig 1: Required PRB for transmission of $1 \mathrm{Mbps}$ as a function of SINR

The virtual load (VL) of cell $c$ can expressed as a function of required resources of all users connected to the serving cell $c$ and the total number of resources $N_{t}$ as follows [15]:

$$
\rho_{c}=\frac{\sum_{u: X(u)=c} N_{u}}{N_{t}}
$$

An overload occurs, when $\rho_{c}>1$, that is when the total required number of PRBs exceeds the total available physical resource in an eNodeB. The degree of overload or otherwise can be expressed using this load parameter. For instance $\rho_{c}=$ 7 , means $1 / 7$ of the users are satisfied.

\subsubsection{Overall State:}

The overall state (OS) is determined using formulations proposed in [16]. Although the virtual load provides the load status of the cell, it is pertinent to know the load status of the cell's environment to avoid Ping-Pong and excessive handovers. The overall state is a parameter that can be used to decide whether an overloaded cell can transfer users and whether a lightly loaded cell can receive users. This will enhance the transfer of users from the most loaded cell to the least loaded. The Overall state is a weighted combination of the load of the cell and the average load of the cell's neighbors. The average load of the neighbor is termed the Environmental State (ES) given by:

$$
E S_{c}=\frac{\left(\rho_{1 c}+\rho_{2 c}+\rho_{3 c}+\cdots+\rho_{n c}\right)}{n}
$$

The overall state can be expressed as a function of the cell load and ES by:

$$
O S_{c}=\mu \rho_{c}+(1-\mu) E S_{c}
$$

The parameter $\mu$ sets the weight of $\rho_{c}$ and $E S_{c}$. The optimal value of $\mu$ was determined to be 0.2 in [16]. Thus, the overall state can be rewritten as:

$$
O S_{c}=0.2 \times \rho_{c}+0.8 \times \frac{\sum_{j=1}^{n} \rho_{j}}{n}
$$

\subsection{Key Performance Indicators}

Load balancing is expected to yield an increase in the overall performance of the network. Some metrics that can be used to evaluate the performance of the network with respect to load balancing include: the number of satisfied/unsatisfied users and the load distribution index of the network. Since a constant bit rate user service is assumed, the throughput will not be a very relevant performance indicator.

\subsubsection{Number of Unsatisfied Users:}

This indicator relates to the number of users (USU) in the cell that can achieve the desired bit rate due to resource limitations. It is desirable to minimize this parameter by transferring load from overloaded cells to other cell that uses the same frequency. The number of unsatisfied user per cell is given by [17]:

$$
Z_{c}=A_{c} \times\left(1-\frac{1}{\rho_{c}}\right)
$$

Where: $A_{c}=$ number of users in the cell and $\rho_{c}=$ virtual load in the cell. Considering the network as a whole, the total number of unsatisfied used is given by:

$$
Z_{n e t}=\sum_{\forall c} \max \left[0, A_{c} \times\left(\frac{1}{\rho_{c}}\right)\right]
$$

\subsubsection{Load Distribution Index:}

The fairness distribution index (LDI) proposed in [18] can be applied for evaluating the fairness of load distribution. Thus, the load distribution index measuring the degree of load balancing of the entire network is given as:

$$
\mu(t)=\frac{\left(\sum_{c} \rho_{c}(t)\right)^{2}}{|N| \sum_{c}\left(\rho_{c}(t)\right)^{2}}
$$

Where $|N|$ is the number of cells in the network (used for simulation) and $t$ is the simulation time. The load balance index $\mu(t)$ takes the value in the interval $\left[\frac{1}{|N|}, 1\right]$. A larger value of $\mu$ indicates a more balanced load distribution among the cells. Thus, the load distribution index is 1 when the load is completely balanced. The aim of load balancing (for CBR users) is to maximize $\mu(t)$ at any time $t$.

\section{DEVELOPMENT OF THE ANFIS MODELS}

In the previous section, two load indicators and two key performance indicators were derived. Based on these parameters, two ANFIS load balancing models can be designed. The first model can be designed using the load indicators and the distribution index as key performance 
indicator. This model is called Load Distribution Index (LDI) model. The second model referred to as unsatisfied user (USU) model, uses the number of unsatisfied users as the KPI and the same load indicators as that of the LDI model. The training, checking and testing data were generated from an open source system level LTE simulator. Different number of users ranging from 5 to 500 was used to generate input-output datasets. Sample of training data used for developing the models are presented in Table 2. The two models proposed are presented in subsequent sub-sections.

Table 2. Sample Training Data for ANFIS Modelling

\begin{tabular}{|c|c|c|c|c|c|c|}
\hline \multirow{2}{*}{ 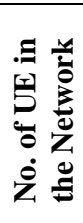 } & \multicolumn{6}{|c|}{ LIs and KPIs for Each eNodeB } \\
\hline & $\frac{n}{0}$ & 5 & $\tilde{0}$ & $\overline{\mathrm{a}}$ & $\stackrel{?}{\mathscr{D}}$ & $\underset{D}{2}$ \\
\hline \multirow{4}{*}{5} & $\begin{array}{c}\mathrm{eNB} \\
1\end{array}$ & $\begin{array}{c}0.014 \\
7\end{array}$ & $\begin{array}{c}0.023 \\
8\end{array}$ & $\begin{array}{c}0.928 \\
3\end{array}$ & 0 & 1.4784 \\
\hline & $\begin{array}{c}\text { eNB } \\
2\end{array}$ & $\begin{array}{c}0.026 \\
4\end{array}$ & $\begin{array}{c}0.017 \\
1\end{array}$ & $\begin{array}{c}0.617 \\
0\end{array}$ & 0 & 2.6495 \\
\hline & eNB & 0.015 & 0.024 & 0.939 & 0 & 1.5803 \\
\hline & & & & & & \\
\hline \multirow{6}{*}{100} & eNB & 0.290 & 0.469 & 0.928 & 0 & 29.043 \\
\hline & & 4 & 4 & 2 & & \\
\hline & eNB & 0.520 & 0.336 & 0.616 & 0 & 52.056 \\
\hline & 2 & 5 & 4 & 9 & & \\
\hline & eNB & 0.307 & 0.477 & 0.937 & 0 & 30.754 \\
\hline & 3 & 5 & 9 & 9 & & \\
\hline \multirow{6}{*}{120} & eNB & 0.333 & 0.566 & 0.914 & 0 & 33.307 \\
\hline & 1 & 0 & 7 & 9 & & \\
\hline & eNB & 0.625 & 0.391 & 0.609 & 0 & 62.547 \\
\hline & 2 & 4 & 5 & 9 & & \\
\hline & eNB & 0.375 & 0.575 & 0.941 & 0 & 37.597 \\
\hline & 3 & 9 & 5 & 5 & & \\
\hline \multirow{6}{*}{200} & eNB & 0.561 & 0.952 & 0.916 & 0 & 56.184 \\
\hline & 1 & 8 & 2 & 0 & & \\
\hline & eNB & 1.048 & 0.659 & 0.610 & 3.806 & 104.80 \\
\hline & 2 & 0 & 0 & 9 & 8 & \\
\hline & eNB & 0.638 & 0.966 & 0.944 & 0 & 63.863 \\
\hline & 3 & 6 & 1 & 3 & & \\
\hline \multirow{6}{*}{300} & eNB & 0.858 & 1.425 & 0.921 & 0 & 85.872 \\
\hline & 1 & 7 & 1 & 4 & & \\
\hline & eNB & 1.563 & 0.999 & 0.614 & 44.71 & 156.39 \\
\hline & 2 & 9 & 7 & 5 & 4 & \\
\hline & eNB & 0.948 & 1.440 & 0.943 & 0 & 94.866 \\
\hline & 3 & 6 & 9 & 4 & & \\
\hline
\end{tabular}

\begin{tabular}{|c|c|c|c|c|c|c|}
\hline \multirow{4}{*}{450} & eNB & 1.329 & 2.127 & 0.930 & 32.71 & 132.94 \\
& 1 & 4 & 2 & 7 & 1 & \\
\cline { 2 - 7 } & eNB & 2.333 & 1.530 & 0.620 & 105.7 & 233.34 \\
& 2 & 4 & 2 & 0 & 1 & \\
& & & & & & \\
\cline { 2 - 7 } & eNB & 1.422 & 2.151 & 0.944 & 142.2 & 142.27 \\
& 3 & 7 & 3 & 4 & 7 & \\
& & & & & & \\
\hline
\end{tabular}

\subsection{LDI Model}

The inputs were converted into linguistic variables each of which has a triangular-shaped membership function. From experiments, two fuzzified variables (low and high) were found to be most suitable for this model. There are 3 inputs each with 2 fuzzy variables, thus we have a total of 8 rules for the knowledge base. The rules, which were written using the ANFIS rule editor Graphic User Interface (GUI), are given in Fig.2. The Model is made up of 34 nodes, 8 linguistic rules, 8 linear parameters and 18 non-linear parameters. Thus, the total number of parameters is 26 . In order to achieve good generalization, the number of input-output pairs should be several times greater than the total number of parameters being evaluated. 84 training data pairs were used for training, which makes the ratio of data points to number of parameters to $84 / 26=3.2$. The structure of the LDI ANFIS model is shown in Fig.3. The training goal of 0.087541 was reached in two training epochs.

The proposed model was validated using checking and testing data sets. Model validation is a two-fold process of ensuring that the model does not over fit the data and to test the model for data that was not used for training. The checking and testing data were also extracted from the simulator using different number of UEs (randomly chosen in the rage 4-400). The checking dataset is used in training to prevent model over fitting of the data by using an early stopping technique. When the checking data is used, the model parameters that correspond to the minimum checking data model error are selected. The testing data set are not used in the training but are used to check the degree to which the model predicts the corresponding data set output values. When data checking was implemented, a checking data model error of 0.184051 against the training data model error of 0.0875408 was obtained. An average testing error of 0.096799 was obtained for the testing data set. The test results for checking and testing validation are shown in Fig 4 and 5 respectively.

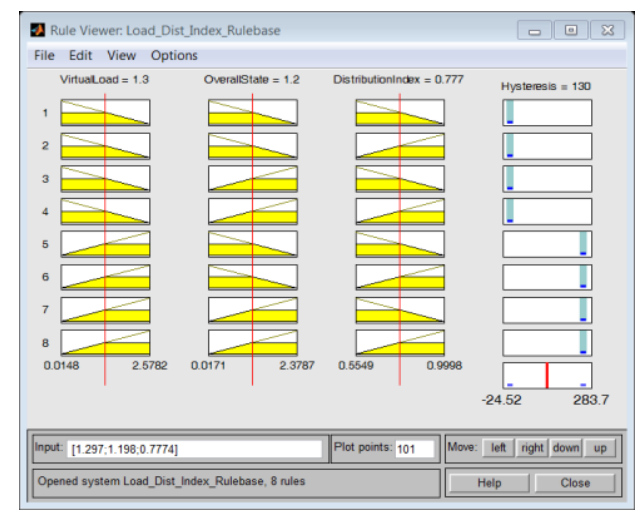

Fig 2: Rule Viewer for LDI Model 


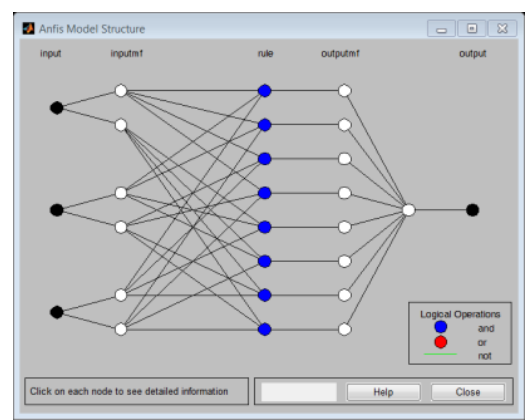

Fig 3: Load distribution Index Load Balancing ANFIS Model Structure

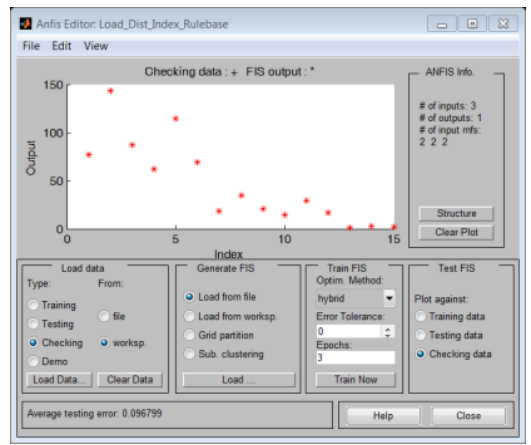

Fig 4: Model Validation of checking data set for LDI ANFIS Model

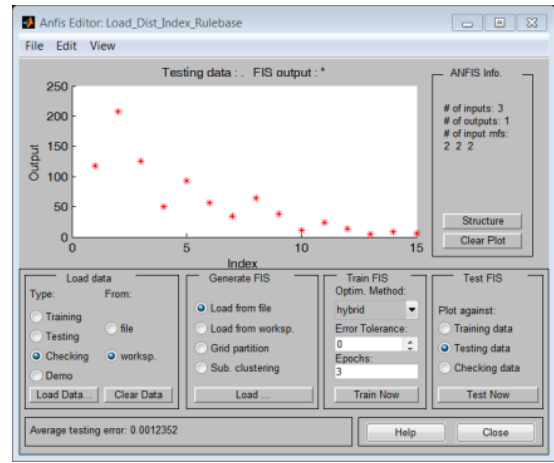

Fig 5: Model Validation of testing data set for LDI ANFIS Model

\subsection{USU Model}

As earlier mentioned, the inputs to this model are the virtual load (VL), Overall State (OS) and the number of unsatisfied users. These three crisp inputs are fuzzified using 2 triangular membership functions for each input. The knowledge base of the model is then generated after which fine-tuning of parameters using hybrid-learning algorithm is implemented to set up the model. The fuzzy reasoning is illustrated in Fig.6.

The model parameters are similar to those of the LDI. The model contains 34 nodes, 8 linguistic rules, 8 linear parameters and 18 non-linear parameters. Thus, the total number of parameters is 26 and the ratio of data points to number of parameters is 3.2. The structure of the USU ANFIS model is shown in Fig. 7. The training goal of 0.0662014 was reached in two training epochs.

The model validation gives a checking data model error of 0.179228 against the training data model error of 0.0662014 was obtained. An average testing error of 0.00067149 was obtained for the testing data set. The test result for checking and testing validation are shown in Fig 8 and 9 respectively.

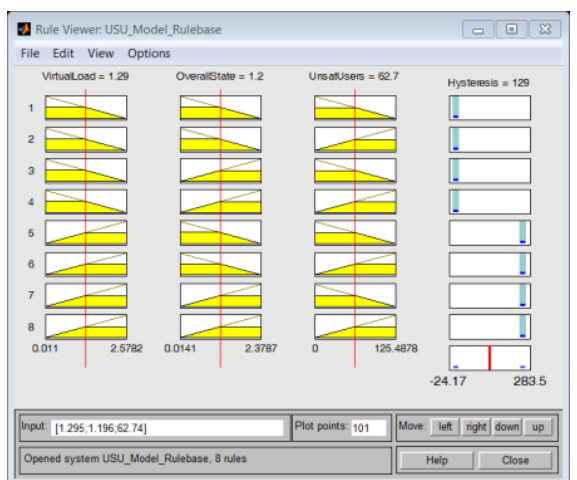

Fig 6: Rule Viewer for USU Model

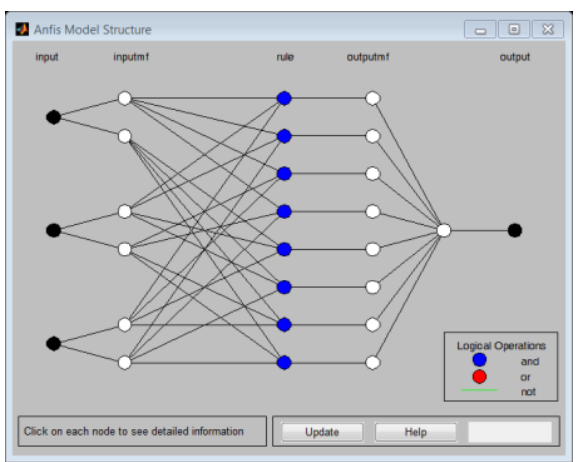

Fig 7: Load distribution Unsatisfied User ANFIS Model Structure

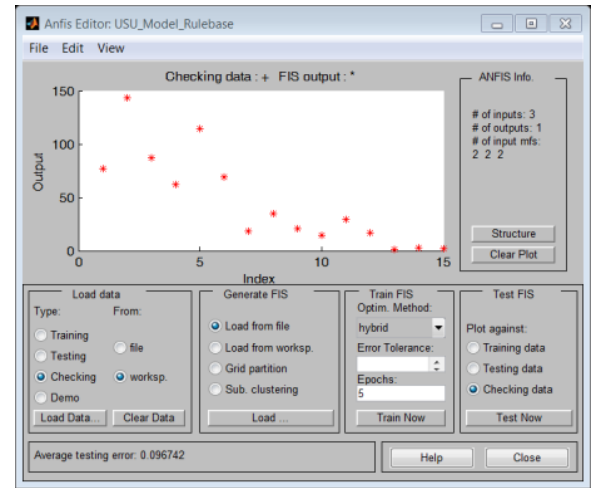

Fig 8: Model Validation of checking data set for USU ANFIS Model

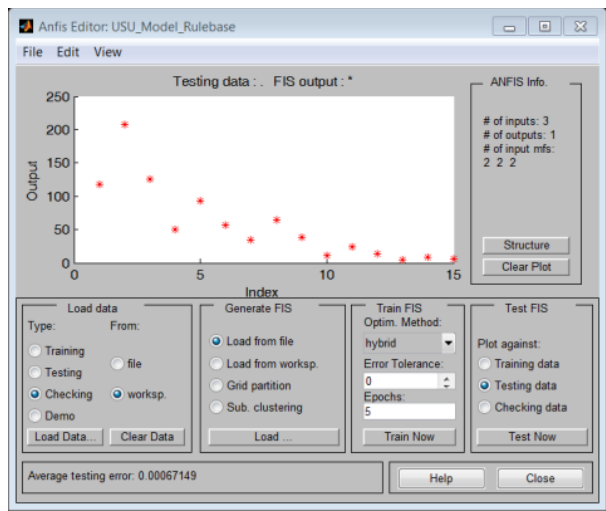

Fig 9: Model Validation of testing data set for USU ANFIS Model 


\section{SIMULATION RESULTS AND DISCUSSION}

For the LDI Model, increment of hysteresis with the virtual load is linear with a gradient value of 100 and starts from the origin (Fig. 10). These relationships indicate that the virtual load, which is the main parameter to be controlled, plays a key factor in determining the hysteresis. When the hysteresis value is high, then more loads are transferred from a particular eNodeB. The overall state of the eNodeB is a load parameter that considers the load of its neighbors as well as its own load. This is necessary in order to avoid excessive and Ping-Pong handovers when the neighbor are also highly loaded. Thus, it is desired that when the overall state is high, load balancing should be limited by reducing the hysteresis value (Fig. 11). The primary aim of load balancing in the LDI model is to optimize the load distribution index. In other words, when the distribution index is low, then the hysteresis should be increased and conversely when the distribution index is high, the hysteresis should be reduced. This objective is achieved in the LDI model as shown in Fig. 11.

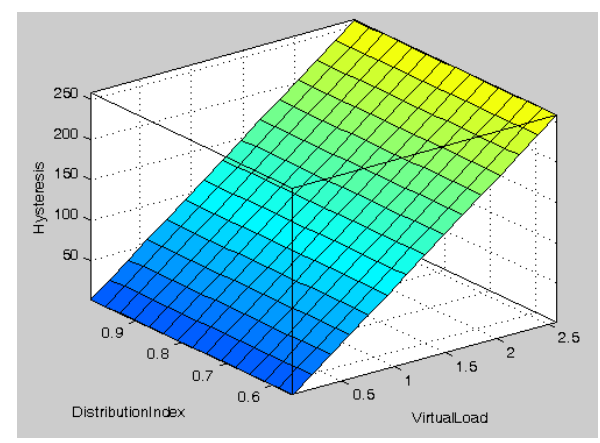

Fig 10: LDI Model - Impact LDI and VL on hysteresis value

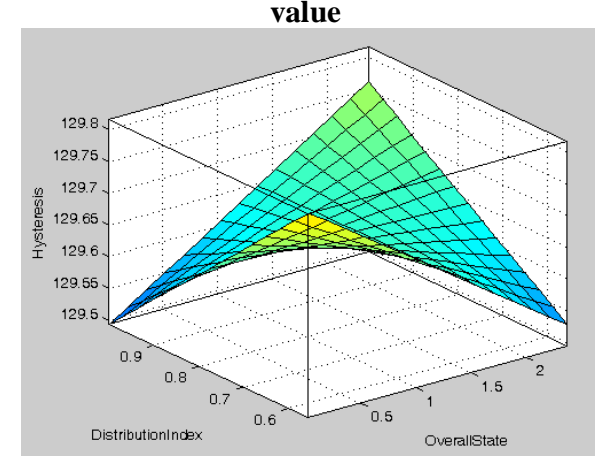

Fig 11: LDI Model: Impact LDI and OS on hysteresis value

The simulation ${ }^{1}$ results for the USU model are presented in Fig. 12 and Fig. 13. As in the case of the LDI model, the increment of hysteresis with the virtual load is linear with a gradient value of 100 and starts from the origin (Fig. 12). Similarly, an increase in the overall state tends to decrease the hysteresis since it considers the load status of other neighboring eNodeBs (Fig. 13). The fundamental aim of load balancing in the USU model is to minimize the number of unsatisfied user. Thus, when the number of unsatisfied users increases, the probability of load balancing should be increased by increasing the hysteresis value (Fig. 13). The number of unsatisfied parameters begins to contribute to the

\footnotetext{
${ }^{1}$ All Matlab m-files used in the simulations reported are available in
} Bibliography 1. system when an overload occurs (value of virtual load greater than 1).

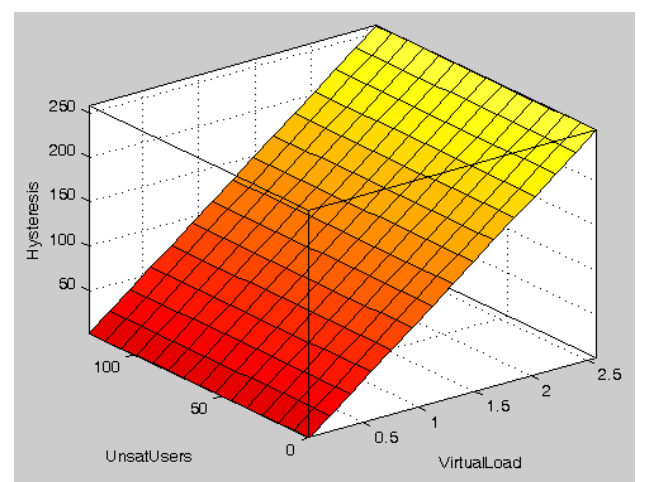

Fig 12: USU Model - Impact USU and VL on hysteresis

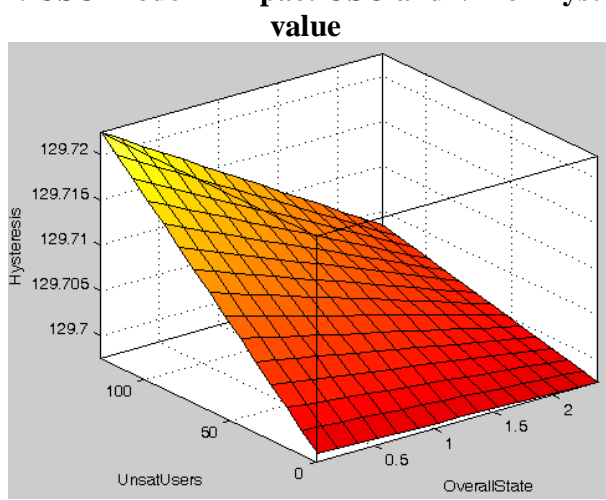

Fig 13: USU Model - Impact OS and USU on hysteresis value

\section{CONCLUSION}

In this work, a new paradigm for dynamic load balancing in 3GPP LTE based on neural encoded fuzzy models is presented. This new approach is informed by the need to leverage on human intuition and tolerance for uncertainty and imprecision in mobile computing. The approach also ensures that unnecessary and Ping-Pong handovers are avoided by ensuring a more informed load balancing. This is necessary considering the fact that handover is a computationally demanding task. Two different ANFIS models were proposed, each corresponding to a key performance indicator. The first model was premised on fairness of load distribution among the cells. The objective of this model is to optimize the load distribution among cells. Thus, the larger the load distribution index, the fairer distribution of load among cells. To achieve this objective, the KPI is used as an input to the ANFIS model so that the best cell hysteresis value can be obtained. The KPI is used as an input alongside load indicators that cause the distribution index to vary. Two load indicators were used, namely: Virtual load and overall state. The virtual load is the degree of overload or otherwise of the serving cell. It is the principal load parameter that indicates when load balancing should be initiated. The overall state of the eNodeB weighs the load conditions of the serving cells and its neighbors to determine whether it can accept or transfer loads. This model is termed, LDI Model. The second model is based on the number of unsatisfied users as a key performance indicator. Here, the objective is to minimize the number of unsatisfied users in the network. The numbers of unsatisfied users in the cell are then used as an input to the ANFIS model that determines hysteresis value for load balancing. As in the case of the LDI model, the virtual load and overall state are also used as inputs. This model is referred to as the USU model. 


\section{REFERENCES}

[1] Chandrasekhar V., Andrews J., and Gatherer A., 2008. "Femtocell networks: a survey," IEEE Communications Magazine, Vol. 46, No.9, pg.59-67.

[2] Cheolhee Park et al. 2012. "LTE-advanced modem design: challenges and perspectives," IEEE Communications Magazine, Vol. 50, No.2, pg. 178-186

[3] Radio-Electronics. 2012. Self-Organizing Networks [online]. Available: http://www.radio-electronics.com.

[4] 3GPP TS 36.300 version, 2011. "LTE; Evolved Universal Terrestrial Radio Access (E-UTRA) and Evolved Universal Terrestrial Radio Access Network (EUTRAN); Overall description; Stage 2", Technical Specification, version 10.4.0 Release 10

[5] Stefania Sesia, Issam Toufik and Matthew Baker, 2009. "LTE - The UMTS Long Term Evolution: From Theory to Practice", $1^{\text {st }}$ edition, John Wiley \& Sons, Ltd. , West Sussex, UK.

[6] ETSI TR 136 902, 2010. "LTE; Evolved Universal Terrestrial Radio Access Network (E-UTRAN); Selfconfiguring and self-optimizing network (SON) use cases and solutions," Technical Specification, version 9.0.0 Release 9.

[7] Andreas Lobinger, Szymon Stefanski, 2010. Thomas Jansen and Irina Balan, "Load Balancing in Downlink LTE Self-Optimizing Networks," IEEE $71^{\text {st }}$ VTC 2010, Taipei, Taiwan.

[8] Hao Wang et al. 2010."Dynamic Load Balancing in 3GPP LTE Multi-Cell Networks with Heterogenous services”, ICST Conference, Beijing.

[9] H. Wang, 2010. "Dynamic Load Balancing and Throughput Optimization in 3GPP LTE Networks", IWCMC 2010, Caen, France.

[10] Manfred R., Jakob B., Paul A., and Wilfried W., 2009. "Ruled-based Algorithms for Self-x Functionalities in Radio Access Networks," Conference Proceedings of ICT-Mobile Summit.

[11] Rodriguez J., de la Bandera I., Munoz P., and Barco R., 2011. "Load Balancing in a Realistic Urban Scenario for LTE Networks," IEEE $73^{\text {rd }}$ Vehicular Technology Conference (VTC Spring), pp. 1-5

[12] Klaus Wehrle, Mesut Gunes and James Gross, 2010. "Modelling and tools for Network Simulation," SpringerVerlag, Germany, pg. 173-190.

[13] ETSI TR 136 211, 2012. "LTE; Evolved Universal Terrestrial Radio Access (E-UTRA); Radio Frequency (RF) system scenarios," Technical Report Version 8.2.0, Retrieved Feb., 20, 2012, from http://www.3gpp.org.

[14] Aderemi A. Atayero and Matthew K. Luka, 2012. “Adaptive Neuro-Fuzzy Inference System for Load
Balancing In 3GPP LTE," (IJARAI) International Journal of Advanced Research in Artificial Intelligence, Vol. 1, No. 1, pp. 11-16.

[15] Andreas Lobinger, Szymon Stefanski, 2010. Thomas Jansen and Irina Balan, "Load Balancing in Downlink LTE Self-Optimizing Networks," IEEE $71^{\text {st }}$ VTC 2010, Taipei, Taiwan.

[16] Lin Zhang et al. 2011. "A Two-layer Mobility Load Balancing in LTE Self-Organization Networks," $13^{\text {th }}$ IEEE Conf. on Information Technology, pp.925-929.

[17] Ingo Viering, Andreas Lobinger and Szymon Stefanski, 2010. "Efficient Uplink Modeling for Dynamic SystemLevel Simulations of Cellular and Mobile Networks," EURASIP Journal on Wireless Communications and Networking, pp. 1-15.

[18] R. Jain, D.M Chiu and W. Hawe, 1984. "A Quantitative Measure of Fairness and Discrimination for Resource Allocation in Shared Systems," Technical Report, Digital Equipment Corporation, DEC-TR-301.

\section{BIBLIOGRAPHY}

[1] Matthew K. Luka and A.A. Atayero (2012), Adaptive Neuro-Fuzzy Inference System Models for Dynamic Load Balancing in 3GPP LTE, Lambert Academic Publishing GmbH \& Co. KG, Germany, ISBN: $\quad 978-3-659-19288-3, \quad$ Available: http://qr.net/anfis1.

[2] Aderemi A. Atayero and Matthew K. Luka, "ANFIS Modeling of Dynamic Load Balancing in LTE", Chapter in: Integrated Models for Information Communication Systems and Networks: Design and Development, ISBN 13: 978-14666-220-1, IGIGlobal Publishers, USA, Due Oct. 2012.

[3] Aderemi A. Atayero and Matthew K. Luka, 2012. "Applications of Soft Computing in Mobile and Wireless Communications", International Journal of Computer Applications, IJCA ISSN: 0975-8887, Vol. 45, № 22, pp. 48-54, May 2012.

[4] Aderemi A. Atayero and Matthew K. Luka, 2012. “ A Soft Computing Approach to Dynamic Load Balancing in 3GPP LTE", International Journal of Computer Applications, IJCA ISSN: 0975-8887, Vol. 43, № 19, pp. 35-41, Apr. 2012.

[5] Aderemi A. Atayero, et al. 2011. "3GPP Long Term Evolution: Architecture, Protocols and Interfaces", International Journal of Information and Communication Technology research IJICT, ISSN: 2223-4985, Vol. 1, № 7, pp. 307-310, Nov. 2011. 